\title{
Le corps transgressif dans le roman La Perte de
} l'image de Peter Handke

Der transgressive Körper im Roman Der Bildverlust oder durch die Sierra de Gredos von Peter Handke

The Transgressive Body in Handke's Novel Der Bildverlust oder durch die Sierra de Gredos

\section{Gauthier Labarthe}

\section{OpenEdition}

Journals

Édition électronique

URL : https://journals.openedition.org/ceg/9991

DOI : $10.4000 /$ ceg.9991

ISSN : 2605-8359

\section{Éditeur}

Presses Universitaires de Provence

Édition imprimée

Date de publication : 20 janvier 2020

Pagination : 163-172

ISBN : 979-10-320-0253-7

ISSN : 0751-4239

\section{Référence électronique}

Gauthier Labarthe, «Le corps transgressif dans le roman La Perte de l'image de Peter Handke », Cahiers d'Études Germaniques [En ligne], 78 | 2020, mis en ligne le 28 octobre 2021, consulté le 31 janvier 2022. URL : http://journals.openedition.org/ceg/9991 ; DOI : https://doi.org/10.4000/ceg.9991 


\title{
Le corps transgressif dans le roman La Perte de l'image de Peter Handke
}

\author{
Gauthier LABARTHE \\ Université Toulouse-Jean-Jaurès, CREG (EA 4151), Toulouse
}

Dans La Perte de l'image ${ }^{1}$, Peter Handke raconte l'histoire d'une jeune femme, une héroïne " princesse de la finance ", qui décide un matin de quitter sa grande ville d'Europe du Nord pour rejoindre la Manche, ce plateau aride situé au centre de l'Espagne et qui fut le théâtre des aventures de Don Quichotte. Elle veut y retrouver l'auteur qu'elle a chargé d'écrire sa biographie, et plus spécialement le séjour qu'elle a effectué quelques années plus tôt dans la Sierra de Gredos. S'amorce alors un voyage qui s'apparente davantage à une errance au cours de laquelle la protagoniste, en rompant avec ses attaches identitaires, se trouve " [enlevée] à l'ordre social, à l'ordre du savoir, à l'ordre des pères, engagé[e] dans un processus de redéfinition de soi incertain " et donc en proie à un " état d'insécurité ontologique ${ }^{2}$ ». La déliaison qui en résulte modifie en profondeur les modalités de perception du réel. Le sujet handkéen se trouve en effet livré à la contingence du monde, à l'hypervisualité et à l'abondance de signes qui l'aliènent et entraînent la perte de toute authenticité. Outre la dépossession de soi-même engendrée par cette " pluie d'étoiles filantes très drue ${ }^{3}$ " qui assaille la conscience, cette expérience dysphorique de la perte de l'image signifie également une ouverture indéfinie aux événements qui embarquent ainsi le sujet handkéen dans le devenir et la fluence d'un monde qui n'est plus normé, c'est-à-dire déterminé par les distances et les directions topographiques, mais qui se caractérise par la seule logique de l'intensité. L'espace et le corps sont démétrisés, affranchis des normes de mesure et n'obéissent plus désormais à une représentation décomposable, quantifiable. Se soustrayant à toute détermination topographique et géographique, cet espace est traversé par des forces, des intensités optiques, tactiles qui révèlent à la banquière la texture et le poids du monde. Autant d'éléments qui le rattachent à l'espace " lisse " tel que le défınissent Gilles Deleuze et Félix Guattari. Il s'agit d'un :

1. Peter Handke, Der Bildverlust oder Durch die Sierra de Gredos, Frankfurt a. M., Suhrkamp, 2002.

2. Lambert Barthélemy, Fictions contemporaines de l'errance : Peter Handke, Cormac McCarthy, Claude Simon, Paris, Classiques Garnier, 2011, p. 28-29.

3. Sauf indication contraire, toutes les traductions se réfèrent à la version française : Peter Handke, La Perte de l'image, traduction de l'allemand par Olivier Le Lay, Paris, Gallimard, 2004 (ici p. 172). "Sternschnuppenfall so dicht auf dicht " (Handke, Der Bildverlust, p. 200). 
espace d'affects, plus que de propriétés. [...] dans le lisse [,] des matériaux signalent des forces ou servent de symptômes. C'est un espace intensif, plutôt qu'extensif, de distances et non pas de mesures. [...] La perception y est faite de symptômes et d'évaluations [...]. C'est pourquoi ce qui occupe l'espace lisse, ce sont les intensités, les vents et les bruits, les forces et les qualités tactiles et sonores ${ }^{4}$.

Dé-métriser l'espace revient donc avant tout à ouvrir l'espace et le corps pour en libérer toutes les potentialités de reconfiguration sensible et esthétique ${ }^{5}$. De ce point de vue, le corps serait transgressif au sens premier et étymologique du terme. Bertrand Westphal nous rappelle en effet que cette notion signifie avant tout un dépassement de la mesure et que "[transgresser] dérive du latin trangredi, dont le sens était à l'origine spatial ", puisque " [chez] les Romains, on transgressait lorsque l'on passait de l'autre côté d'une borne ou d'un fleuve ${ }^{6}$ ". Surtout, le critique français nous explique que la "trangressio était aussi une figure de rhétorique (chez Cicéron), que l'on traduirait aujourd'hui par "hyperbate" " ". À la manière de cette figure consistant à ajouter un élément qui briserait la clôture d'une construction, le corps transgressif décrit un mouvement expansif engendré par une vérité excédentaire qu'il recèle en lui ${ }^{8}$. Cette vérité intime qui repousse et transgresse les frontières n'est cependant pas une plongée dans les profondeurs de l'être et de la terre, mais une dilatation vers les marges, à la manière de ces cristaux qui " ne deviennent et ne grandissent que par les bords, sur les bords ", engagés dans un mouvement qui les fait " non plus s'enfoncer, mais glisser tout le long, de telle manière que l'ancienne profondeur ne soit plus rien, réduite au sens inverse de la surface ${ }^{9}$."

Véritable ruban de Möbius, le corps introduit un type de spatialité marquée par la logique de l'intensité. "L'espace intensif ", pour reprendre le concept deleuzien, désigne alors ce lieu où se tisse la trame des devenirs, embarquant le corps, dans un premier temps, dans une logique régressive. Celle-ci confronte la banquière à l'angoisse existentielle du chaos et surtout de l'altérité du grand Dehors illimité et indéterminé. Ainsi revenu à une forme de préhistoire de son développement, le corps ouvre le texte à l'incarnation. Par une esthétique du pan et par le jeu du proche et du lointain qui restituent au corps sa capacité à être affecté et, partant, ses qualités pathétiques, il orchestre la débâcle du

4. Gilles Deleuze, Félix Guattari, Mille plateaux, Paris, Éditions de minuit, 2002, p. 598.

5. Nous renvoyons notamment à l'ouvrage de Judith Sarfati-Lanter, qui a analysé cette reconfiguration dans le roman Mon année dans la baie de Personne. Judith Sarfati-Lanter, Donner forme au sensible : la perception dans l'œuvre de Peter Handke, Malcolm Lowry et Claude Simon, Paris, H. Champion, 2013.

6. Bertrand Westphal, La Géocritique : réel, fiction, espace, Paris, Éditions de Minuit, 2007, p. 72.

7. Ibid.

8. Il faut voir dans ce phénomène le prolongement et le renouvellement de certaines problématiques présentes dans les œuvres des années 1980 et 1990. En effet, comme nous l'explique Fabjan Hafner, ce qui distingue la production handkéenne à partir des années 2000 des deux décennies précédentes, c'est notamment la volonté de reconstruire des filiations, qui se traduit par une dynamique du " retour ", de la "revenance " dont le tracé dessine des lignes de fuite qui ouvrent l'espace à de nouvelles potentialités. Fabjan Hafner, Peter Handke : Unterwegs ins neunte Land, Wien, P. Zsolnay, 2008.

9. Gilles Deleuze, Logique du sens, Paris, Éditions de Minuit, 1969, p. 19. 
système représentationnel propre aux arts mimétiques. Délogé de son ancrage sémantique, il subit une excentration qui l'entraîne dans la mouvance des devenirs et des dynamiques métamorphiques qui brouillent les frontières entre les êtres et les différentes catégories. S'établit alors un " consensus rythmique ${ }^{10}$ " entre l'individu et le monde permettant de restaurer une relation œcuménique perdue jusque-là dans le fond des âges. Exhumant des décombres une rythmicité archaïque, ancestrale et originelle, parce que synchrone avec la pulsation du monde, le sujet s'élargit aux dimensions où il se perd dans la splendeur de l'anonymat.

\section{Esthétique de la surface et régression}

Face à la mouvance d'un monde livré au chaos de l'indéterminé, Handke recourt à un mode d'approche du réel emprunté à Cézanne. Privilégiant alors une esthétique de la surface et du pan, Handke redonne au réel sa texture et sa corporéité. Démétriser l'espace nécessite avant tout de déconstruire les catégories mathématiques qui gouvernent sa représentation et celle des corps. L'esthétique du pan qu'il développe de plus en plus à partir de La Leçon de la Sainte-Victoire ${ }^{11}$ permet de saisir au plus près les mouvements tectoniques de la réalité. Réutilisant la méthode cézanienne d'appréhension du réel, Handke restitue à l'écriture une lisibilité du réel qui opère par strates et par pans. Cet art de la surface, en rompant avec les codes traditionnels de représentation propres aux arts mimétiques, donne à l'écrivain les outils pour exprimer la réalité d'un corps en fluence permanente et embarqué par la mobilité d'un monde insaisissable. En effet, si, comme l'écrivait Erwin Panofsky, la perspective moderne " constitue un fragment de monde [...] à l'intérieur duquel corps et espace libre commencent, étant établi qu'ils sont d'égale valeur, à passer pour les deux formes d'expression d'une unité homogène et indivisible ${ }^{12}$ ", la perspective cézanienne, immédiate en ce qu'elle repose sur la proximité au réel, diffère de l'espace mis en place par la perspective mathématique et qui ne serait ni infıni, ni homogène, ni continu, mais psychophysiologique.

Comme nous l'explique Deleuze, nous assistons à un véritable changement de paradigme représentationnel. Partant du postulat formulé par Valéry selon lequel " [c]e qu'il y a de plus profond en l'homme, c'est la peau ${ }^{13}$ ", le philosophe

10. Edward Twitchell Hall, La Danse de la vie : temps culturel, temps vécu, traduit de l'anglais (États-Unis) par Anne-Lise Hacker, Paris, Seuil, 1984, p. 11.

11. Dans ce texte, Handke raconte l'expérience ainsi que les enseignements qu'il a pu tirer de son ascension de la montagne Sainte-Victoire. Outre l'apprentissage d'une nouvelle vision, il y décrit la découverte d'une nouvelle spatialité dans laquelle le sujet aurait accès à l'unité du Sensible. Voir notamment Arlette Camion, "Peter Handke, une question de regard ", Germanica 10, 1992, p. 155-168.

12. Erwin Panofsky, La Perspective comme forme symbolique, traduction de l'allemand sous la direction de Guy Ballangé, Paris, Éditions de Minuit, 1975, p. 114.

13. Paul Valéry, L'Idée fixe, Euvres II, Paris, Gallimard (coll. " La Pléiade »), 1960, p. 215-216. 
français constate que " tout remonte à la surface ${ }^{14}$ ". Derrière cette poussée vers l'épiderme, vers la membrane que constitue le corps en tant qu'interface avec le milieu extérieur, se révèle le mouvement souterrain du devenir et du paradoxe qui apparaît justement comme " destitution de la profondeur, étalement des événements à la surface, déploiement du langage de cette limite ${ }^{15}$ ". Pour affronter et parcourir ce monde, le sujet handkéen doit dès lors recourir à de nouvelles pratiques cheminatoires. Ainsi la banquière se retrouve-t-elle confrontée, dès le début de son voyage dans la Sierra de Gredos, à un paysage ravagé par un ouragan. Elle se voit obligée de recourir à une " allure " (Gehen) " étrange " et " merveilleuse " pour dépasser la vision apocalyptique de ce lieu, " son regard [n'ayant] pas osé mesurer l'étendue du désastre ${ }^{16}$ " :

\begin{abstract}
Kein Weg, der nicht versperrt war von Bäumen oder deren auseinandergebrochenen Trümmern. Man mußte drüber hinwegklettern, unten durchschlupfen oder sie langwierig umgehen - wobei gleich das nächste Hindernis zu umgehen war, und gleich noch eins, so daß man vielleicht unversehens wieder hinaus aus dem Wald geriet. So entschied sie sich für das Klettern und Kriechen. [...] Aber was gab es an dieser Zerstörung denn überhaupt zu betrachten? Die Zerstörung hat kein Geheimnis, so dachte auch sie bei ihrem ersten Waldgang nach dem Jahrtausendsturm. Erst in den Wiederholungen gingen ihr dann die Augen auf. Mit den entwurzelten Bäumen war zugleich eine mächtige Masse des Wurzelgrunds von zuunterst nach oben gekehrt worden. Es waren das annähernd Halbkugeln oder auch Pyramiden aus Sand, Lehm und Steinbrocken, senkrecht gekippte Querschnitte, wo aus dem Mittelpunkt strahlenförmig die am Rand dann gekappten und zerfleddert in die Luft vorstoßenden Seitwärtswurzeln weggingen, während die Mitte von einem ungleich dickeren, dem Betrachter entgegenragenden Wurzelbruchstück, sozusagen der Mutterwurzel, gebildet wurde ${ }^{17}$.
\end{abstract}

Face à un paysage où s'accumulent les décombres des arbres renversés, empêchant ainsi toute progression physique, la banquière met en pratique une vision de surface qui lui permet de dépasser l'illusion réaliste d'un secret caché dans les profondeurs de la réalité et d'agencer ainsi un ensemble de formes géométriques qui, en plus de renverser les catégories spatiales traditionnelles, dédouble l'image qui s'offre à elle dans le jeu de la répétition des différences. Par ce biais, Handke choisit la disparition de la ligne, incapable de cerner de l'intérieur et de manière adéquate le centre organisateur de la scène - la " racine-mère " - qui donc garantirait dans l'espace de représentation l'unité des corps. En s'orientant vers une peinture inspirée de la Flächenkunst, et donc en privilégiant la contiguïté de la surface picturale, Handke fait du contour, ordinairement conçu comme le principe même de la forme individuelle, une limite qui devient instable et ambiguë - le regard suit les racines qui, dans leur mouvement latéral, s'échappent hors-cadre, dans les marges et dans l'air -, entre deux corps, deux êtres, deux événements, incapable d'exprimer autre chose que le tremblement à la surface ou à la jointure entre deux éléments. Ce n'est jamais le même spectacle qui

14. Deleuze, Logique du sens, p. 17.

15. Ibid., p. 18.

16. Handke, La Perte de l'image, p. 61. "Oder hatte der Blick nur davor zurückgescheut, sie alle mit einem Mal aufzunehmen ?" (Handke, Der Bildverlust, p. 65).

17. Handke, Der Bildverlust, p. 65. 
s'offre à la banquière lorsqu'elle vient à cet endroit, il s'y rajoute toujours de nouvelles singularités et de nouvelles différences qui enrichissent la réalité ${ }^{18}$. Maintien illusionniste du rapport entre figure et fond, la ligne du contour fait basculer les conditions mêmes de l'identité.

Dans ce passage, la dialectique de perméabilité montre la perturbation des rapports entre fond et figure, entre espace et figure, dévoilant par ce biais un processus de régression au sens freudien du terme, c'est-à-dire au sens de Rückbildung, de retour à l'image sensorielle d'où la représentation, au sens de Vorstellung, était sortie. Le télescopage des pans de réalité, parcourus par le regard mais aussi vécus à même le corps du sujet handkéen, montre, dans le tourbillon des devenirs, une nature en train de faire et de défaire les distinctions entre les êtres. À travers le recours à un art pictural cézannien, Handke semble ici affirmer un art de la composition, d'une coexistence et coprésence physiques, voire une synchronie par friction, mélange et contact, entre réalités hétérogènes. Synchronies de l'image qui, quant à elle, exigerait ce qu'on pourrait appeler une iconologie matérielle et qui devrait toucher le spectateur et son regard, finalement métamorphosé. La sensation, désagrégée dans le processus régressif, se trouve restituée à sa matérialité première et originaire, sa Rohmaterialität.

\section{Le corps pathétique}

Pour arriver à ce résultat, l'esthétique du pan ne suffit pas et Handke met en place une véritable heuristique corporelle de la réalité : si c'est bien toujours le regard qui lit et décrit la réalité, c'est en revanche le corps qui l'explore, procédant ainsi à une véritable archéologie du toucher. Pour ce faire, l'auteur opère un passage de l'optique à l'haptique. En effet, ce terme, du verbe grec aptô (toucher), ne désigne pas une relation extrinsèque de l'œil au toucher, au sens où on ne peut dissocier l'œil du toucher, mais une " possibilité du regard ", un type de vision distinct de l'optique : le réel est tâté du regard, conçu pour être vu de près, et, comme l'écrit Henri Maldiney, " dans la zone spatiale des proches, le regard procédant comme le toucher éprouve au même lieu la présence de la forme et du fond ${ }^{19}$ ". Cette prééminence du corps et de la matière au premier plan fait appel à une vision de tout près, qui s'abolit comme point de vue. Le corps se fait tactile, incarnation visuelle, c'est-à-dire un matériau dans lequel viennent s'inscrire d'autres images et de nouvelles formes visuelles qu'il n'est possible d'activer que par la création d'autres regards qui disloquent, fragmentent la perception sensorielle du monde. Ces autres regards, ce sont ceux des êtres qui habitent cette forêt dévastée, ces " animaux dont la présence était surprenante ici ", qu'il s'agisse des écureuils qui " n’avaient pas été exterminés " et qui " restés dans leur retraite, [...] zigzaguaient à présent le long des troncs ", des lièvres qui,

18. C'est ce qu'elle constate avant de décrire la scène : " Mit jedem neuen Eindringen jedoch wirkte die Zerstörtheit gewaltiger ", puis, quelques lignes après : "Und trotzdem jedesmal wieder zusätzliche, wie eben, über Nacht, niedergebrochene Bäume, Äste, Kronen. » Ibid., p. 65.

19. Henri Maldiney, Regard, parole, espace, Paris, Éditions du Cerf, 2012, p. 195. 
" d'ordinaire si farouches, filaient à découvert d'un gîte à l'autre ${ }^{20}$ ", ou bien encore des arbres dotés d'une vie, d'une force mouvante. La banquière disparaît en tant que foyer central de la description et de la perspective au profit d'une vision à même de se déplacer en " escaladant " ou en " rampant ".

En effet, si l'image dépeinte dans la peinture traditionnelle reposait sur l'effet illusoire des jeux de surfaces bidimensionnelles produits par la fausse profondeur, l'image décrite ici refuse de limiter le spectateur à un point de vue unique, qui lui permettrait de dominer visuellement le champ pictural. Au contraire, Handke prend le contre-pied d'une telle organisation perspectiviste par la mise en place de dispositifs qui achèvent d'ébranler la logique représentationnelle et figurative. L'auteur entend créer l'illusion de pouvoir tourner autour de l'image elle-même pour la découvrir sous une multitude de points de vue différents qui ouvrent le corps à sa qualité proprement pathétique. Ainsi dépossédé de son ancrage sémantique, le corps assure le passage de l'optique à l'haptique et recouvre par ce biais la capacité d'être affecté et d'accueillir des forces mouvantes extérieures qui déplacent les figures représentées, les sortent de leur forme déterminée et délimitée. Les corps retrouvent alors leur puissance expressive et se voient redonner vie et mouvement, à l'image de ces arbres qui, " [bien] que le vent fût très doux " et " sans crier gare ", " étaient en train de tomber [...], les uns très lentement, les autres au contraire très brusquement ${ }^{21} "$ :

Der eine Riese neigte sich, fast sachter Ruck um Ruck, bis an seiner Stelle nur noch, ohne Aufprallaut, der Luftraum war, samt flimmerndem Nachbild der Äste; dem anderen splitterte plötzlich die schwere Krone ab; dem dritten zog es in einem Augenblick den Boden weg; und bei all dem, einander wie aus sämtlichen Waldecken antwortenden, Bersten und Krachen herrschte zwischendrin eine vollkommene Stille; auch vom Wind kein Geräusch ${ }^{22}$.

Le jeu dialectique du proche et du lointain, qui se traduit ici par la délégation de la focale, concrétise le renversement non seulement des catégories, mais également de la relation proxémique que la banquière porte à son paradoxe. Ainsi explique-t-elle que " [si elle s'est] rapprochée si près, c’[est] précisément pour être enfin inaccessible ", " pour éviter que [ses] ennemis ou [ses] adversaires ne puissent [...] lever la main sur [elle] ${ }^{23}$ ", avant de révéler qu'elle n'agit ainsi que par peur de la mort. La protagoniste exprime ici un paradoxe que Lacan a formulé en ces termes :

Il y a là une horrible découverte, celle de la chair qu'on ne voit jamais, le fond des choses, l'envers de la face, du visage, les sécrétats par excellence, la chair dont tout sort, au plus profond même du mystère, la chair en tant qu'elle est souffrante, qu'elle est informe,

20. Handke, La Perte de l'image, p. 54.

21. Handke, La Perte de l'image, p. 65. "Trotz des nur schwachböigen Windes kam es im Umkreis aus heiterem Himmel zu einem teils gemächlichen, teils jähen [...] Stürzen " (Handke, Der Bildverlust, p. 70).

22. Handke, Der Bildverlust, p. 70-71.

23. Handke, La Perte de l'image, p. 142. "So nah bin ich nur gekommen, um unnahbar zu werden ", "So [...] daß meine Feinde oder Gegenspieler auch nicht einmal einen Finger gegen mich rühren konnten. " Ibid., p. 163. 
que sa forme par soi-même est quelque chose qui provoque l'angoisse. Vision d'angoisse, identification d'angoisse, dernière révélation du tu es ceci - Tu es ceci qui est le plus loin de toi, ceci qui est le plus informe ${ }^{24}$.

L'angoisse profonde et existentielle qu'éprouve la banquière devant l'altérité du Dehors trouve une illustration parfaite lors de sa rencontre charnelle avec un compagnon de voyage dans une tente alors qu'ils campaient près de Pedrada. Cette scène se construit à partir du contraste entre l'homme " allongé sur son lit comme sur une civière ", semblable à un mort ${ }^{25}$, et la banquière animée par le désir et la vie :

In dem Maße, wie der Mann eingefallen war und eine zunehmende Auflösung darstellte, blühte die Frau an seiner Seite auf. [...] [S]ämtliche ihrer Formen weiteten, spannten und streckten sich. Sie als ganze gewann an Volumen, vergrößerte und straffte sich, und wurde zugleich schwer und schwerer - warm schwer, schön schwer. [...] Die Schenkel, neben dem kläglichen Fast-Gerippe, hoben, buckelten und wölbten sich, ebenso wie die Brüste; der Mund, das Umkehrbild zu dem kadaverhaften des Mannes, stand leicht geöffnet und ließ die Spitze der Zunge sehen $[\ldots]^{26}$.

Pour comprendre cette scène, il faut considérer l'anomisme de l'espace environnant plongé dans la nuit, envahissant les êtres d'une présence impalpable qui déforme les contours, brouille l'identité des êtres qui ne parviennent plus à s'individualiser. La " dissolution croissante " du réel engendre alors " l'image inversée " d'un monde qui n'est plus perceptible que dans la spectralité d'une présence impalpable et indéfınissable. En suivant la métamorphose de ce corps, nous pénétrons un monde régi par une logique de l'anti- et anté-catégoriel, qui fait saillir les perturbations de la matière sous la forme et ébranle profondément les déterminations. Il faut en déduire, avec Bachelard, que sur le plan du détail, "Pensée et Réalité apparaissent comme déliées et l'on peut dire qu'en s'éloignant de l'ordre de grandeur où nous pensons, la Réalité perd en quelque sorte sa solidité, sa constance, sa substance. En résumé, Réalité et Pensée sombrent ensemble dans le même néant ${ }^{27}$."

De même que le Laocoon représentait un corps crispé et contracté montrant, selon Goethe, "le mouvement et sa cause " dans la "double action " (doppelte Handlung ${ }^{28}$, exprimée par chaque figure avec une intensité contorsionnée, nous observons ici un être qui met en scène ensemble et en même temps les sources intérieures et leurs effets, les affections psychiques et leurs expressions motrices. Apparait alors un être dépersonnalisé par un milieu vibratoire où extériorité et intériorité, dehors et dedans se confondent en une seule et même substance. Dans son texte "Sur le Laocoon », Goethe situait la source de l'action convulsive de la figure dans l'intériorité, dans la psyché de l'individu dont le corps devenait

24. Lacan, Le Séminaire, II. Le moi dans la théorie de Freud et dans la technique de la psychanalyse, Paris, Seuil, p. 186. C'est Lacan qui souligne.

25. Handke, La Perte de l'image, p. 374 ; " auf dem Bett ausgestreckt wie auf einer Bahre und schien tot " (Handke, Der Bildverlust, p. 440).

26. Ibid.

27. Gaston Bachelard, Essai sur la connaissance approchée, Paris, J. Vrin, 1969, p. 253.

28. Johann Wolfgang Goethe, Schriften zur Kunst und Literatur, Stuttgart, Reclam, 1999, p. 111. 
alors une surface d'affection sur laquelle le dehors et l'illimité venaient déposer une empreinte pathétique. Nous retrouvons dans La Perte de l'image un semblable jaillissement de pathos qui s'incarne dans une image irréelle mais puissante de la hantise et de la folie. S'il y a donc expressivité de l'image, elle doit être envisagée comme contact matériel entre la psyché et le milieu, en tant que correspondance palpable entre intérieur et extérieur, que sympathie indicielle entre fond et forme.

La perspective immédiate se transforme en un vaste miroir aux résonances où le sujet s'efface devant le vécu sensuel, sensoriel ou émotif. Le règne de l'indice s'ouvre lorsque se substitue la présence immédiate à la représentation : l'espace devient pathétique et laisse place, par les déplacements qu'il orchestre, à l'imagination du vivant. Seul un corps métamorphique, capable de transformations semble à même de saisir le monde dans ses différentes mutations. Aberrant et malléable, le corps excédentaire qui se déforme sous nos yeux fait en effet l'objet d'une transmutation permettant d'accéder à la " plus sensuelle des sensations ${ }^{29}$ ", au point de transformer les individus en " vases communicants ${ }^{30}$ ". De ce point de vue, la transgressivité libère le corps de la stase en faisant émerger les potentialités de renouveau et de liberté contenues dans un espace qui, jusque-là, restait captif de son identité forclose et imperméable à l'altérité. La révolution des arts mimétiques sert à explorer et rebaptiser un monde. Comme si cette nouvelle image avait pour but de rendre le vide visible, le matériel immatériel, le corporel incorporel. Le corps se trouve ainsi étroitement lié au thème de la puissance : la corporéité propre au " Nouveau Monde " dévoile en quelque sorte des corps d'avant le monde.

\section{La métamorphose}

À travers le corps se jouent donc des procédés de déplacements, de condensation, voire de dramatisation qui produisent un spectre d'identité au long duquel, dans les franges, se répartit le " je ". La voix qui résonne d'écho en écho fonde une parole qui, telle la Chose héraclitéenne et présocratique, nourrit la dialectique du déterminé et de l'indéterminé. En plus du jeu de substitutions qu'elles mettent en place, les métamorphoses du sujet d'écriture bouleversent les codes de l'identité et de l'écriture de soi en niant tout principe de vérité, de causalité, et nous obligent à ne plus penser en termes de contradictions ni d'oppositions, mais de juxtaposition ou de succession. À travers le parcours régressif de la protagoniste sur les terres de son enfance, l'auteur met en place un espace où les formes peuvent s'échanger et se métamorphoser. Handke met en scène des corps saturés, à la limite de leurs capacités sensorielles et qui oscillent en permanence entre les deux extrêmes du trop-plein et de l'évidement. Mais plutôt que de demeurer dans l'aller-retour entre l'euphorie et la panique, l'angoisse, il trouve une issue

29. Handke, La Perte de l'image, p. 371; " zu der sinnlichsten der Sinnlichkeiten " (Handke, Der Bildverlust, p. 435).

30. Ibid., p. 263 ; « kommunizierende Gefäße ” (ibid., p. 308). 
dans la porosité entre le genre humain et non-humain. Il fait entrer en résonance la vie animale, minérale et la vie historique, existentielle des hommes, au point que les frontières entre les règnes s'amoindrissent : l'intimité du corps se lie alors à l'immensité du monde. Pour ce faire, Handke s'emploie tout d'abord à élargir l'individu aux dimensions de l'univers. Le mouvement d'expansion qui porte le corps à la rencontre du monde puis à sa fusion avec l'univers trouve son expression dans le processus métamorphique, permettant de penser une identité en mouvement, en transit entre plusieurs identifications possibles, ou pour le dire autrement, une identité nomade.

La fin du roman porte la régression au comble des puissances métamorphiques, emportant alors le récit dans un jeu de variations destituant la banquière de sa propre identité. Celle-ci accède à un état impersonnel et surtout pré-individuel synonyme de retour au stade préhistorique du sujet, que produit, entre autres, la " tension de toutes les cordes de son corps" ${ }^{31}$ ". C'est donc une femme métamorphosée que nous voyons sortir de la grotte dans laquelle elle se trouvait :

\footnotetext{
Was für ein Durcheinander wieder, bis sie endlich oben auf dem Weg war. Erst einmal kroch sie auf allen vieren, krabbelte, robbte, wie arm- und beinlos, auf dem Bauch, schlurfte seitwärts, ungeschickt, jap lump [...]

\begin{abstract}
Schon vorher, im Sichaufrappeln, hatte sie einen Schrei ausgestoßen, nein, ein Röcheln, ein Gestammel aus bloßen Konsonanten. Und jetzt kam ein Bellen, ein stockheiseres, aus der Schönen heraus, einem wie halb im Schlamm, einem Wolfsheulen, wie nicht zu ihr gehörig, hinter dem Horizont hervor, einem Altmännerhusten, einem Mischton aus Muhen, Blöken und Meckern, dem bald tatsächliche Tierlaute aus der Weite zu antworten schienen, hier ein Hähergratter, dort das Flöten einer Nachtigall, dort das Pfeifen eines königlichen Milans, schriller als jede Schiedsdrichterpfeife ${ }^{32}$.
\end{abstract}

Le traitement que réserve Handke à la métamorphose peut être éclairé par la théorie deleuzienne des " devenirs ", des concepts de "devenir-animal ", " devenir-fou " ou "devenir imperceptible ". Ainsi le philosophe cherche-t-il à penser le sens comme un " événement " plutôt que comme " état de chose ". Le devenir échappe à toute identité fixe et forclose en transformant le corps en un seuil où s'effectuent les passages d'un état à un autre, mais sans pour autant que le sujet se confonde totalement avec ces états. On le voit ici, à aucun moment la banquière, malgré le devenir régressif qui l'affecte à et la fait revenir à un état animal, presque larvaire, ne s'identifie complètement aux différents animaux auxquels elle est assimilée. Cela s'explique par le fait que le " sujet larvaire " évoque une individuation ouverte aux devenirs. Devenant toujours autre chose que ce qu'il est, l'homme devient quelque chose d'inhumain, engagé dans un processus travaillé par le mythe, sans que ce phénomène ne soit réductible à une imitation des animaux. Nous retrouvons le corps modelé par le tracé de devenirs qui prennent et mélangent différentes formes, comme nous l'explique Deleuze :

Les devenirs-animaux ne sont pas des rêves ou des phantasmes. Ils sont parfaitement réels. Mais de quelle réalité s'agit-il ? Car si le devenir-animal ne consiste pas à faire

31. Traduction par nos soins. " Anspannen aller Körpersehnen " (Handke, Der Bildverlust, p. 728).

32. Ibid., p. 728-729. 
l'animal ou à l'imiter, il est évident aussi que l'homme ne devient " réellement " autre chose. Le devenir ne produit pas autre chose que lui-même. C'est une fausse alternative qui nous fait dire : ou bien l'on imite, ou bien l'on est. Ce qui est réel, c'est le devenir lui-même, le bloc de devenir, et non pas des termes supposés fixes dans lesquelles passerait celui qui devient ${ }^{33}$.

Les rectifications qu'apporte le narrateur handkéen à sa description ou bien encore les comparaisons qui ponctuent le passage cité plus haut ancrent ces lignes dans une démarche de déréalisation où se signale encore la présence d'une conscience assistant au spectacle d'une natura naturans emportée dans un délire de devenirs et de formes dont on observe, à travers les déictiques spatio-temporels, la processualité à l'œuvre. Ces éléments sont surtout les indicateurs d'une noncoïncidence de la forme et du fond, inhérente au travail métamorphique du devenir. En effet, la métamorphose se caractérise avant tout par sa fonction métaphorisante qui, selon l'étymologie et conformément à la première opération du travail de la figure, renvoie à la translatio, au déplacement. Ce que Freud - dans le domaine du rêve, du mot d'esprit et du symptôme - désignait comme un incessant " travail du déplacement " (Verschiebungsarbeit) se vérifie ici dans la puissance qu'ont les figures d'excéder la représentation habituellement marquée par des valeurs descriptives, dénotatives ou même narratives. De ce point de vue, il apparaît que la régression ne se définit plus selon une perspective involutive, mais qu'elle doit être envisagée comme quelque chose qui « fait appel à des déplacements de lieux et des dyschronies ${ }^{34}$ " et qui nous donne à voir dans l'informe, en tant que mouvement de régression, non pas l'" abjection " mais " l'excentration " " qui dépossède le corps de ses ancrages [...] sémantiques".

L'espace intervallaire révèle toute la positivité de la neutralité qui renverse dialectiquement les catégories corporelles : l'évidement suscite le remplissage, voire le trop-plein, créant alors un espace que l'on peut décrire comme l'espace à la fois de la potentialité et de l'absence. Mouvant et protéiforme, le sujet d'écriture caméléon s'approprie certaines caractéristiques de la pensée mythique, dont la singularité tient justement à sa grande malléabilité ainsi qu'à sa propension à se répéter dans la différence. Il se révèle être un foyer d'échos privilégiant l'équivocité, à l'image d'un sens introuvable et cependant central qui, par son " épaisseur sémantique ", " déborde de toutes parts la linéarité du signifiant " et qui transforme le sujet d'écriture en un "palais des miroirs dans lequel chaque mot renvoie en tous sens à des significations cumulatives ${ }^{35}$ ".

33. Deleuze et Guattari, Mille plateaux, p. 291.

34. Pierre Fédida, Par où commence le corps ? Retour sur la régression, Paris, Presses Universitaires de France, 2000, p. 12.

35. La comparaison est de Jacques Soustelle, cité par Gilbert Durand, Les Structures anthropologiques de l'Imaginaire, Paris, Dunod, 1981, p. 413. 


\section{Rythmicité du « chaosmos»}

Par son pouvoir métamorphique, le sujet d'écriture se mue en cellule germinale à partir de laquelle se déploie le jeu des substitutions, révélant une logique de prolongement/ dépassement au cœur des relations entre le sujet d'écriture, les personnages et les objets du monde, dévoilant alors la nature mythique d'un sujet transfiguré dans ses simulacres. L'enjeu essentiel des lignes citées plus haut et de toute métamorphose, est, selon la formule de l'auteur, de " rendre inconnu le connu ; arpenter l'espace de l'inconnu et l'agrandir ${ }^{36}$ ". Il s'agit de prendre la mesure du monde et de s'affranchir de la pesanteur des choses. Aux prises avec l'indéterminé, le sujet d'écriture se déploie, se déplace et se transmue dans le texte à travers la " multiplicité des résonances ${ }^{37}$ " qui se déploient en orbes, pour s'élever peu à peu aux dimensions de l'univers et atteindre enfin un sentiment d'harmonie et de paix. Ce qui se joue à travers la perspective immédiate, c'est donc la place qu'occupe le corps au sein de cet espace. Cette place, on l'a vu, est synonyme d'ouverture et d'excentration, mais une excentration qui porte le corps à la rencontre du monde et qui a pour principal effet d'affirmer par et dans le corps une échelle première. Il s'agit de raconter l'espace concret dont chacun occupe le centre et d'ancrer ainsi la géographie intime de l'individu à l'intérieur de la géographie du monde. Pour cela, il faut donner, dans le texte, une dimension corporelle à la matière de l'espace. Fonder la réalité humaine en retrouvant, en redécouvrant son appartenance, son ancrage au monde, tel est le principal enjeu de la rythmicité corporelle. L'appréhension matérielle de l'espace permet à Handke de transmettre une expérience à la fois intime et anonyme : une sensation d'espace fondatrice de toute aperception de soi.

Le principal résultat de ce jeu du proche et du lointain, en plus de conjurer l'angoisse originelle, réside dans une véritable révolution du corps et de la relation proxémique : c'est désormais l'espace qui gravite autour du corps qui lui confère à la fois une mesure et lui imprime un rythme. L'excentration $d u$ corps produite par les dispositifs mimétiques orientés vers l'haptique a déjà mis en valeur la scansion du corps et du toucher qu'imposait la nouvelle logique du sens. Il convient maintenant d'articuler l'infime particule spatiale que représente le corps à l'échelle immense et infınie du monde afın de mieux cerner le " consensus rythmique " qui s'établit entre l'individu handkéen et le milieu avec lequel il interagit. Ce lieu où devient possible le partage de l'espace par et dans le corps, Handke l'ébauche à la fin du roman, lorsqu'il conduit la banquière à Hondareda, ville imaginaire située dans les marges de la réalité et qui se caractérise notamment par son rythme. Ce " rythme d'Hondareda ${ }^{38}$ " ne signifie pas seulement un déplacement physique, mais embrasse également l'ensemble des gestes qui composent la vie sociale et quotidienne de ce peuple,

36. Peter Handke, Die Wiederholung, Frankfurt a. M., Suhrkamp, 1986, p. 262 : « Bekanntes unbekannt machen; den Bereich des Unbekannten abschreiten und vergrößern ".

37. Roger Caillois, Le Mythe et l'Homme, Paris, Gallimard, 1987, p. 30.

38. "Hondareda-Rhythmus" (Handke, Der Bildverlust, p. 746). 
désenfouissant par ce biais d'anciennes et archaïques formes de vie. Il s'agit pour ces individus de refonder la possibilité de "l'être total " qui ne peut s'exprimer que dans un contre-rythme, un contre-mouvement qui infuse toutes les couches de la vie quotidienne. Alors que la banquière éprouvait à tel moment l'expérience du corps fragmentaire, elle parvient, par le rythme, à rentrer en résonance avec l'intimité du monde :

Ich kann nichts Besseres für dich tun, als bei dem zu bleiben, was ich tue. Indem ich das, was ich tue, rhythmisch tue, gewissenhaft rhythmisch, ohne Nachlässigkeit, immer weiter den mir entsprechenden Rhythmus erfüllend und ihn zum Vibrieren, Oszillieren und Vorspuren bringend, tue ich für mich und für dich das Beste, was ich für mich wie für dich tun kann. " - "Was ist für Sie Rhythmus? " (so der Autor dann). Sie: "Bestärkung des Vorhandenen ${ }^{39}$.

Dans le roman, le contraste est fort entre les figures souvent masculines réduites à un corps de pierre, douloureux et renvoyant davantage à une dépouille, et les corps " intensifiés ", comme nous avons déjà pu le voir avec la scène d'amour. Dans ces scènes, le corps féminin se voit gorgé de vie, du poids de la terre : il se fait Leib. Handke choisit ces moments pour distinguer le corps en tant que Körper et en tant que Leib. Cependant, il dépasse les distinctions habituelles entre ce que l'on pourrait appeler en français (pour reprendre la terminologie de Merleau-Ponty) " corps vivant " (Körper) et " corps propre " (Leib) dans la mesure où ce dernier, bien loin de fonder l'ancrage ontologique et existentiel de l'être-au-monde, signifie plutôt une dépossession. Il est un corps exproprié, transgressif en cela qu'il est " [sorti] par húbris de son espace pour entrer dans un corps étranger ${ }^{40} "$.

Cependant, à travers le rôle joué par la pulsion dans l'essence du rythme, ce sont surtout les processus énergétiques à l'œuvre dans le corps rythmique et rythmé qu'il s'agit de mettre en lumière. La rythmicité du corps apparaît tout d'abord comme un moyen de faire face au chaos du monde et de sa perception. Cette idée est déjà présente et incarnée par l'auteur que la banquière engage pour écrire son roman. Celui-ci, décrit comme un " explorateur de formes ", un " homme versé dans l'art des rythmes ${ }^{41}$ ", reprend la figure, chère à Handke, du géologue-arpenteur qui fouille dans les strates de la réalité la présence de formes afın d'échapper au désastre de l'informe et du chaos. Handke approfondit ensuite avec les Hondarederos sa conception du rythme. Elle se traduit tout d'abord par la pratique chorégraphique qui exhume des décombres du monde des rythmes premiers et originaires. Il s'agit donc d'une échelle qui permet d'épouser la mouvance du monde. Le corps éprouve la fluence du monde en rejoignant son devenir par sa puissance métamorphique, justifiant une conception de la métamorphose comme " mouvement immobile ${ }^{42}$ ". La confusion des milieux

39. Ibid., p. 699.

40. François Hartog, Le Miroir d'Hérodote : essai sur la représentation de l'autre, Paris, Gallimard, 2001, p. 487.

41. Handke, La Perte de l'image, p. 19 ; " der Formenforscher und Rhythmenmensch " (Handke, Der Bildverlust, p. 17).

42. Pierre Brunel, Le Mythe de la métamorphose, Paris, J. Corti, 2003, p. 125. 
qui en résulte se manifeste alors dans une rythmicité du corps, dans une " pulsation existentielle qui [...] déploie notre corps en monde sur la terre, et qui simultanément [...] reploie le monde en notre chair ${ }^{43} "$.

La dialectique pulsative, par son retentissement dans le corps du sujet, accentue la brisure de l'individu, mais produit en même temps l'élargissement de son être aux dimensions de l'univers. Confronté à " cet horrible en dedans-en dehors qu'est le vrai espace ${ }^{44}$ ", le sujet voit se confondre intimité et immensité. En ce sens, le rythme est une énergie reposant sur la cadence dynamique d'une tension et d'une détente, et une énergie productrice du réel. Handke, à travers ce peuple, ne nous fournit pas une représentation d'un monde clos et achevé préexistant à l'œuvre, mais l'événement d'une forme en train de naître par cette dynamique du rythme. Dans un univers décimé où le seul mode d'être au monde est la survie, le rythme s'impose donc comme "la riposte des milieux au chaos " en libérant les énergies créatrices qu'il contient.

Les milieux sont ouverts dans le chaos, qui les menace d'épuisement ou d'intrusion. Mais la riposte des milieux au chaos, c'est le rythme. Ce qu'il y a de commun au chaos et au rythme, c'est l'entre-deux, entre deux milieux, rythme-chaos ou chaosmos [...]. C'est dans cet entre-deux que le chaos devient rythme, non pas nécessairement, mais a une chance de le devenir. Le chaos n'est pas le contraire du rythme, c'est plutôt le milieu de tous les milieux. Il y a rythme dès qu'il y a passage transcodé d'un milieu à un autre, communication de milieux, coordination d'espaces-temps hétérogènes ${ }^{45}$.

Ce milieu intermédiaire, c'est justement le corps par lequel s'opère un partage du réel et des sens. Les Hondarederos recréent par ce biais une relation œcuménique avec le milieu environnant puisqu'ils donnent à voir " la planète entière - ce qu'on appelait jadis l'œcoumène, les terres habitées, et j'avais alors la conviction de n'être plus seul, mais rattaché aux autres, solidaire ${ }^{46}$ ". Ils ne sont pas enracinés dans un territoire ou dans une représentation qui calque celui-ci sur un mètre conventionnel, une norme stable. Ils occupent un seuil et illustrent en cela le principe de transgressivité, qui consiste à occuper un entredeux pénétré de toutes les forces qui naissent et s'expriment à la charnière entre les mondes et les corps. Le corps remplit donc bien sa fonction de frontière ici, mais la conception même de frontière a quelque peu bougé. Il s'agit d'un corps qui serait non seulement limen et limes, mais aussi et surtout un pli par lequel l'intérieur et l'extérieur se rabattraient l'un sur l'autre pour n'être plus que le miroir renversé de l'autre. Pli du milieu intérieur et du milieu extérieur, il serait alors non pas tant une personne, un individu, qu'une singularité pré-

43. Augustin Berque, Écoumène : introduction à l'étude des milieux humains, Paris, Belin, 2016, p. 402.

44. Henri Michaux, Nouvelles de l'étranger, Paris, Mercure de France, 1952, p. 91. Cité par Gaston Bachelard, La Poétique de l'espace, Paris, Presses Universitaires de France, 2012, p. 195.

45. Deleuze et Guattari, Mille plateaux, p. 384-385.

46. Handke, La Perte de l'image, p. 623; " gab einem den gesamten Erdkreis zu sehen - das, was früher Ökumene, die bewohnte Welt geheißen hat, und damit die Überzeugung von Zusammengehörigkeit " (Handke, Der Bildverlust, p. 744). 
individuelle, une individuation non personnelle qui se pousse au milieu pour créer un "supplément de monde ${ }^{47}$ ».

\section{Conclusion}

La représentation et la perception du corps chez Handke est indissociable d'une expérience de la communauté. À la manière de ce que Van Gennep avait déjà mis au jour dans le rite ${ }^{48}$, le corps subit une forme de rite sacrificiel par lequel, après un premier mouvement transgressif synonyme de détachement, de séparation par rapport à ses attaches identitaires, il assure la fondation d'une communitas, c'est-à-dire d'une expérience collective transcendant les différences sociales et ouvrant sur une phase d'agrégation, de regain de sens. Cette " autre corporéité " qu'incarnent les Hondarederos dépossède le sujet handkéen de son propre corps, le déplace aux marges du monde, dans les plis du réel. Au bout du déploiement sphérique se trouve le paradoxe inhérent à la logique du sens régissant l'espace intensif : celui d'un corps incorporel. Ce paradoxe expose l'idéal d'un corps qui délaisse son enveloppe charnelle pour se fondre dans la mouvance du monde. Nous assistons dans ces passages à une abstraction et une purification du corps qui n'est plus qu'élan, impulsion. Il incarne le paradoxe d'une représentation qui valorise la corporéité et la plénitude, l'accomplissement du corps afın de mieux évider le sujet désormais réduit à une pellicule sans volume, une fine membrane poreuse qui, en tant que véritable corps-frontière, longe la surface du monde pour accéder à l'incorporel de l'événement et du devenir.

47. "Zusatz-Welt " (ibid., p. 589). Traduction par nos soins.

48. Arnold Van Gennep, Les Rites de passage : étude systématique des rites de la porte et du seuil, de l'hospitalité [...], Paris, Picard, 1991 [Réimpr. de l'édition de 1909]. 